\title{
Association between a polymorphism in the carboxyl ester lipase gene and serum cholesterol profile
}

\author{
Sara H Bengtsson-Ellmark ${ }^{1}$, Jeanette Nilsson ${ }^{*}, 1$, Marju Orho-Melander ${ }^{2}$, Kerstin \\ Dahlenborg $^{1}$, Leif Groop ${ }^{2}$ and Gunnar Bjursell ${ }^{1}$ \\ ${ }^{1}$ Department of CMB/Molecular Biology, Box 462, Göteborg S-405 30, Sweden; ${ }^{2}$ Department of Endocrinology, Malmö \\ University Hospital, Lund University, Malmö S-205 02, Sweden
}

Carboxyl ester lipase (CEL) is involved in the hydrolysis and absorption of dietary lipids, but it is largely unknown to what extent CEL could be involved in determining the serum lipid levels. The C-terminal part of CEL consists of a unique structure with proline-rich $O$-glycosylated repeats of 11 amino-acid residues each. The common variant of the human CEL gene contains 16 proline-rich repeats, but there is a high degree of polymorphism in the repeated region. While the biological function of the polymorphic repeat region is unknown, it has been suggested that it may be important for protein stability and/or secretion of the enzyme. Given that the polymorphism in the repeated region may affect the functionality of the protein, this study aimed to investigate whether the number of repeated units is correlated to serum lipid phenotype. Comparison of CEL repeat genotype and serum lipid phenotype revealed an association between the number of repeats and serum cholesterol profile. Individuals carrying at least one allele with fewer than the common 16 repeats had significantly lower total and low-density lipoprotein (LDL) cholesterol levels compared to individuals carrying two common alleles. This gives support to the notion that CEL may be involved in determining the plasma lipid composition.

European Journal of Human Genetics (2004) 12, 627-632. doi:10.1038/sj.ejhg.5201204

Published online 28 April 2004

Keywords: CEL; lipase; repeats; polymorphism; cholesterol; LDL

\section{Introduction}

Carboxyl ester lipase (CEL), also referred to as bile salt stimulated lipase (BSSL) or bile salt stimulated cholesterol esterase, is a lipolytic enzyme with a wide substrate specificity that can hydrolyse cholesteryl esters, mono-, di-, and triacylglycerol, phospholipids and esters of fatsoluble vitamins. ${ }^{1}$ CEL is a secretory enzyme primarily expressed at high levels in the lactating mammary gland and the exocrine pancreas, and it is activated by bile salts in the intestine. CEL constitutes up to $5 \%$ of the enzymatic

*Correspondence: Dr J Nilsson, Göteborg University, Department of CMB/Molecular Biology, Box 462, Göteborg, S-405 30, Sweden.

Tel: + 46-31-7733879; Fax: + 46-31-7733801;

E-mail: jeanette.nilsson@molbio.gu.se

Received 30 January 2004; revised 4 March 2004; accepted 18 March 2004 content of the pancreatic juice ${ }^{2}$ and has been suggested to be involved in hydrolysis and absorption of dietary lipids. Studies of CEL knockout mice show that lack of CEL appears to have no effect on the intestinal absorption of unesterified fat. ${ }^{3,4}$ However, the cholesteryl ester hydrolytic activity is unique to CEL in the digestive tract, and CEL has been suggested to function as a supplementary enzyme to ensure effective hydrolysis of triglycerides and phospholipids. $^{5}$

The C-terminal part of CEL consists of a unique structure with proline-rich $O$-glycosylated repeats of 11 amino-acid residues each. ${ }^{6,7}$ The repeated units are encoded in exon 11 of the CEL gene. ${ }^{8}$ The number of proline-rich repeats varies between species, ranging from three in mouse ${ }^{9}$ to 16 in humans ${ }^{6}$ and 39 in gorilla, ${ }^{10}$ while the salmon CEL gene lacks the repeated region. ${ }^{11}$ The mouse CEL is a $74 \mathrm{kDa}$ 
protein while the human CEL, which is extensively glycosylated across the repeated region, has an apparent molecular mass of $120-140 \mathrm{kDa}^{2,12}$

A variation between individuals has been observed in the size of human CEL proteins secreted from the lactating mammary gland into milk, and while the molecular basis for this was initially not clear, indirect evidence suggested that the difference reside in the region of proline-rich repeats located in the C-terminal region of the protein. ${ }^{13}$ Recent evidence shows that there is indeed a variation in the number of repeats in the CEL gene and that this variation can account for the observed differences in protein size between individuals. ${ }^{14}$ Furthermore, more than $50 \%$ of the examined subjects were carriers of alleles that deviate from the common allele containing 16 repeated units, ${ }^{14}$ indicating that there is a high degree of polymorphism in the repeated region. This diversity in the number of repeats was later confirmed in another study where alleles containing between 13 and 18 repeats were reported. ${ }^{15}$

While the biological function of the polymorphic repeat region is unknown, it has been suggested that it may protect CEL from proteolytic degradation ${ }^{16}$ and that $\mathrm{O}$ glycosylation of the repeated sequences is important for the secretion of the enzyme. ${ }^{17}$ Although the role of CEL in the intestine is to aid in the absorption of dietary lipids, it is still largely unknown to what extent CEL may be involved in determining the serum lipid levels. Given that the polymorphism in the repeated region may affect the functionality of the protein, we set out to investigate whether the number of repeated units is correlated to serum lipid phenotype. We have genotyped a total number of 263 individuals and correlated their CEL repeat genotype to their plasma lipid profile. We confirm the distribution of CEL repeat genotypes reported previously, ${ }^{14,15}$ and we can here report an association between the number of repeats and serum cholesterol profile. Individuals carrying at least one allele with fewer than the common 16 repeats had lower total cholesterol and low-density lipoprotein (LDL) cholesterol levels compared to individuals carrying two common alleles.

\section{Methods Subjects}

DNA extracted from blood samples from individuals participating in the Botnia study ${ }^{18}$ was obtained. The 192 type II diabetic patients were randomly chosen, one patient per family. In all, 192 spouses older than 40 years, with NGT during an oral glucose tolerance test (OGTT), and without known family history of diabetes served as controls. All laboratory specimens were taken after a 12-h overnight fast. Glucose tolerance was assessed by a 75-g OGTT. Diagnosis of diabetes was based on WHO criteria from 1998. Fasting concentrations of serum lipids were measured as described earlier. ${ }^{18}$ All participants gave informed consent and the protocol was approved by local ethics committees. A subset of these groups were genotyped in the present study, which comprise a total of 263 individuals, 138 type II diabetic patients ( 77 females and 61 males) and 125 spouse controls (67 females and 58 males). There was no significant difference with respect to gender or age between the groups in this subset.

\section{PCR genotyping}

The CEL pseudogene, which is present in a tandem arrangement with the CEL gene, ${ }^{8}$ contains a corresponding highly variable repeated region. The repeated region in the pseudogene is highly variable with regard to the number of repeats, ${ }^{19}$ and it is not unreasonable to assume that it displays the same range of variability as the repeated region in the CEL gene. Although the CEL pseudogene is generally believed to contain fewer repeats than CEL, it could still possibly interfere with the detection of CEL alleles of different length. To resolve this potential problem, we have in this study used a unique set of primers that unequivocally distinguishes between the repeated regions in CEL and the CEL pseudogene.

Primers (forward primer: 5'-TGGAGATCACCAAGAAGATGGGCAGCAGC- $3^{\prime}$, located in exon 11; and reverse primer: 5'-CCTGCACCATGATGGTGGCTGCAAGTAAG-3', located downstream of the duplication point in unique sequence, MWG-Biotech AG) were designed on the basis of sequence information from GenBank (Accession number AF072711) to amplify specifically the tandem repeated region of the CEL gene. The reverse primer contained a $5^{\prime}$ HEX label to enable detection in the GELSCAN 2000 system (Corbett Research). PCR reactions contained $25 \mathrm{ng}$ genomic DNA and the HotMaster ${ }^{\mathrm{TM} T a q}$ DNA polymerase (Eppendorf) was used with the addition of betaine to a final concentration of $2 \mathrm{M}$. After an initial denaturation at $96^{\circ} \mathrm{C}$ for $1 \mathrm{~min}$, the following cycle conditions were used: $96^{\circ} \mathrm{C}$ for $1 \mathrm{~min}, 66^{\circ} \mathrm{C}$ for $1 \mathrm{~min}, 72^{\circ} \mathrm{C}$ for $1 \mathrm{~min}$ for 30 cycles followed by an extended elongation for $7 \mathrm{~min}$ at $72^{\circ} \mathrm{C}$.

The HEX-labelled PCR fragments were analysed in the Gel-Scan 2000 real-time gel electrophoresis system on a denaturing 5\% PAGE-Urea gel. GelPro Analyzer (Media Cybernetics) software was used to analyse the results.

\section{Subcloning of PCR fragments and sequencing}

PCR products selected for sequencing were cloned into a TA vector (Promega) and sequenced in both directions using vector-specific universal primers. The $\mathrm{CEQ}^{\mathrm{TM}}$ Dye Terminator Cycle Sequencing kit (Beckman Coulter) was used for the sequencing reactions and the results were analysed using the CEQ $^{\text {TM }}$ 2000XL DNA Analysis System (Beckman Coulter).

\section{Statistical analysis}

Group means are expressed as means \pm SEM and the significance of differences was examined using Student's 
t-test. Allele and genotype distributions in control vs diabetes groups were assessed using Pearson's $\chi^{2}$ analysis. Probability values of $P<0.05$ were considered significant.

\section{Results}

\section{Allele and genotype frequencies}

A total of 263 individuals were genotyped. Of these, 125 were control individuals ( 67 females and 58 males) and 138 were patients with type II diabetes (77 females and 61 males). The most common allele was the one containing 16 repeats and this allele was designated $\mathrm{C}$ (common). Alleles that deviated from the common 16 repeats were designated $\mathrm{C}+\mathrm{I}-$ the number of 'extra' or 'missing' repeats. While alleles shorter than the common allele were quite prevalent $(\sim 30 \%)$, the allele frequency of longer alleles was only approximately $3 \%$ (see Table 1 ). There was no significant difference between the distribution of alleles in the control group compared to the patients with type II diabetes $(P=0.1)$.

The most prevalent genotype (CC, two common alleles) was found in $47 \%$ of the individuals. The second most common allele was the $\mathrm{C}-2$ allele containing 14 repeats, closely followed by the $\mathrm{C}-1$ allele containing 15 repeats. The shortest allele detected in the entire material was $\mathrm{C}-6$, corresponding to an allele with only 10 repeats. Carriers of genotypes consisting of one common allele together with any shorter allele accounted for $39 \%$ of the total number of individuals. Only 22 individuals (8\%) were carriers of two alleles that were shorter than the common allele.

Alleles longer than the common allele were uncommon and were found only in 16 individuals $(6 \%)$, and the most common genotype including one longer allele was the $\mathrm{CC}+1$ genotype. While no genotypes with two longer alleles were found, three individuals were found to have $\mathrm{C}+1$ alleles in combination with $\mathrm{C}-2$ alleles, and one individual was found to have a combination of $\mathrm{C}+3$ and $\mathrm{C}-1$ alleles. These four individuals were excluded from

Table 1 Allele frequencies are shown for the control group, the diabetes group and the combination of both groups

\begin{tabular}{lccc}
\hline Allele & $\begin{array}{c}\text { Allele frequency } \\
\text { (all) (\%) }\end{array}$ & $\begin{array}{c}\text { Allele frequency } \\
\text { (controls) (\%) }\end{array}$ & $\begin{array}{c}\text { Allele frequency } \\
\text { (diabetics) (\%) }\end{array}$ \\
\hline C+3 & 0.2 & 0 & 0.4 \\
C+2 & 0.2 & 0.4 & 0 \\
C+1 & 2.7 & 3.6 & 1.8 \\
C & 69.0 & 71.8 & 66.1 \\
C-1 & 8.9 & 7.7 & 10.9 \\
C-2 & 10.8 & 9.3 & 11.3 \\
C-3 & 6.7 & 6.0 & 7.7 \\
C-4 & 1.1 & 1.2 & 1.1 \\
C-5 & 0.2 & 0 & 0.4 \\
C-6 & 0.2 & 0 & 0.4 \\
\hline
\end{tabular}

further studies, as they would otherwise fall in both categories 'at least one shorter allele' ( $<\mathrm{C}$ genotype, defined as either one common allele in combination with one shorter allele or two shorter alleles) and 'at least one longer allele' (>C genotype, defined as either one common allele in combination with one longer allele or two longer alleles), and therefore occur twice in the comparisons. There was no significant difference between the distribution of the genotypes $\mathrm{CC},<\mathrm{C}$ and $>\mathrm{C}$ in the control group compared to the patients with type II diabetes.

\section{Sequencing of alleles}

The classification of genotypes was based on the length of the fragments observed after PCR amplification of the repeated region (a representative section of a gel is shown in Figure 1), and was verified by sequencing of a selection of PCR products to confirm that the estimated sizes corresponded to the appointed number of repeats. Totally, 526 alleles with sizes varying from 10 to 19 repeats were detected. The PCR product classified as the $\mathrm{C}-6$ allele was shown to contain 10 repeats, a $\mathrm{C}-1$ allele 15 repeats, a $\mathrm{C}-2$ allele 14 repeats and a $\mathrm{C}+3$ allele 19 repeats. The repeats are not entirely identical, and it is therefore possible to a certain extent to distinguish which repeats are missing in the shorter alleles. Hence, sequencing revealed that in the C-6 allele the missing repeats were repeat numbers 9,10 , $11,12,13$ and 14 . In the selected $\mathrm{C}-1$ allele, repeat number 12 was missing and the $\mathrm{C}-2$ sample lacked repeats number 10 and 11. Three extra repeats, corresponding to one additional repeat number 9 and two additional repeats number 10, were inserted between repeats 10 and 11 in the PCR product representing the $\mathrm{C}+3$ allele.

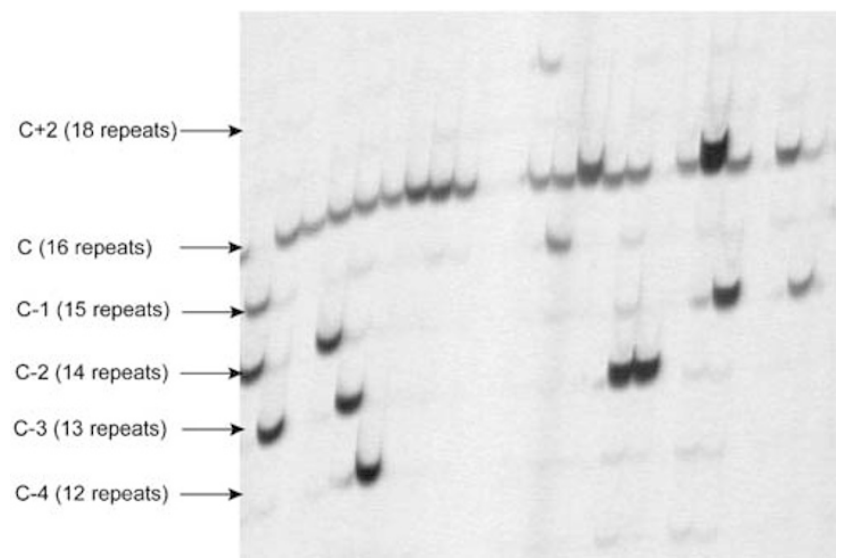

Figure 1 Representative example of a gel used for genotyping of the alleles. Specific PCR primers, one of which was labelled with the fluorescent label HEX, were used to amplify the region containing the repeats. The resulting PCR products were separated on a PAGE-urea gel and visualised using laser detection. 


\section{Comparison of lipid profile between genotypes}

The different parameters included in the study of lipid profiles comprised sex, age, body mass index (BMI), waisthip-ratio (WH), plasma cholesterol, high-density lipoprotein (HDL) cholesterol, triglycerides (TG) and low-density lipoprotein (LDL) cholesterol. Data were missing or incomplete for 29 of the 263 individuals (eight in the control group and 21 in the diabetes group) and hence they were excluded from further studies.

The diabetes group had significantly higher BMI, WH and TG than the control group and also significantly lower HDL, while there were no differences between the groups with respect to total cholesterol or LDL (Table 2). Grouping according to genotype such that the first genotype group contained all individuals homozygous for the common allele (CC genotype) and the second group contained all individuals with at least one allele shorter than the common allele ( $<\mathrm{C}$ genotype) did not reveal any statistically significant differences in either the diabetes or the control group (Table 3), and power analysis indicated that a larger study group would be required. However, for total cholesterol and for LDL, the same trend, with lower total cholesterol and LDL in the $<\mathrm{C}$ group, was observed in both diabetics and controls. Given that total cholesterol and LDL levels did not differ between the diabetes group and the control group (Table 2) and that there is an equal distribution of alleles, as well as CC, $<\mathrm{C}$ and $>\mathrm{C}$ genotypes, in the two groups, they were merged for the analysis of genotype influence on total cholesterol and LDL levels. A comparison of the total plasma cholesterol level between the CC $(n=112)$ and the $<\mathrm{C}(n=108)$ genotypes in the merged group showed that the $<\mathrm{C}$ genotype group had significantly lower levels of total plasma cholesterol than the CC genotype group (CC $6.2 \pm 0.1$ vs $<\mathrm{C}$ $5.8 \pm 0.1 \mathrm{mmol} / \mathrm{l}, P=0.044$ ) (Figure $2 \mathrm{a}$ ). While no reliable statistical data could be obtained for the group with at least one longer allele due to the low number of subjects in that group ( $>\mathrm{C}, n=10$ ), the average total plasma cholesterol appeared to be higher than that for the CC group $(6.4 \pm 0.5 \mathrm{mmol} / \mathrm{l})$ (Figure 2a). Analysis of LDL levels showed that the $<\mathrm{C}$ genotype carriers had significantly lower LDL plasma level $(3.9 \pm 0.1 \mathrm{mmol} / \mathrm{l})$ compared to the CC genotype carriers $\quad(4.3 \pm 0.1 \mathrm{mmol} / \mathrm{l}) \quad(P=0.045)$ (Figure 2b). Similar to the observation for total cholesterol levels, the group with at least one longer allele had higher LDL $(4.6 \pm 0.6 \mathrm{mmol} / \mathrm{l})$ than the CC group (Figure 2b), although this could not be statistically ascertained.

\section{Discussion}

The first evidence that the number of repeats in the human CEL gene varies between individuals came from a study by Strömqvist and co-workers on the apparent molecular mass of the CEL protein secreted into milk. ${ }^{13}$ They found a variation in the size of CEL proteins that could not be explained by differences in carbohydrate content, and indirect evidence suggested that the difference resides in a region of repeats located in the C-terminal region of the protein. ${ }^{13}$ Following up on this, Lindquist et $a l^{14}$ recently reported that there is indeed a variation in the number of repeats between individuals. Their study of a restriction polymorphism within exon 11 of the CEL gene showed that as many as $56 \%$ of the study subjects were carriers of alleles with a number of repetitions that varied from the common 16 repeat allele. Direct sequencing of two of the

Table 2 Comparison of gender distribution, age, BMI, WH, total cholesterol, HDL, TG and LDL between the diabetes and the control groups

\begin{tabular}{lccccccc}
\hline Group & Age (years) & BMI & WH & Cholesterol (mmol/l) & $H D L(m m o l / l)$ & $T G(\mathrm{mmol} / \mathrm{l})$ & $L D L(\mathrm{mmol} / \mathrm{l})$ \\
\hline Diabetes & $61.8 \pm 0.9$ & $28.6 \pm 0.5$ & $0.92 \pm 0.01$ & $5.95 \pm 0.11$ & $1.08 \pm 0.03$ & $1.90 \pm 0.10$ \\
Control & $60.5 \pm 0.8$ & $26.6 \pm 0.4$ & $0.88 \pm 0.01$ & $6.04 \pm 0.13$ & $1.27 \pm 0.03$ & $1.39 \pm 0.07$ & $4.02 \pm 0.10$ \\
& & $P<0.01$ & $P<0.01$ & & $P<0.01$ & $P<0.01$ & 0.12 \\
\hline
\end{tabular}

$P$-values are indicated where statistically significant differences were found.

Table 3 Comparison of gender distribution, age, BMI, WH ratio, total cholesterol, HDL, TGs and LDL between the CC and $<\mathrm{C}$ genotypes in the diabetes and control groups

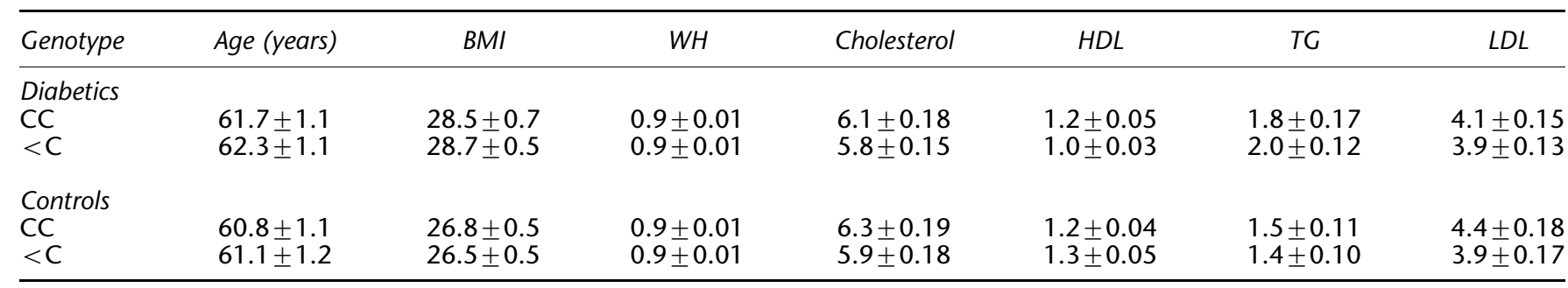


a

cholesterol

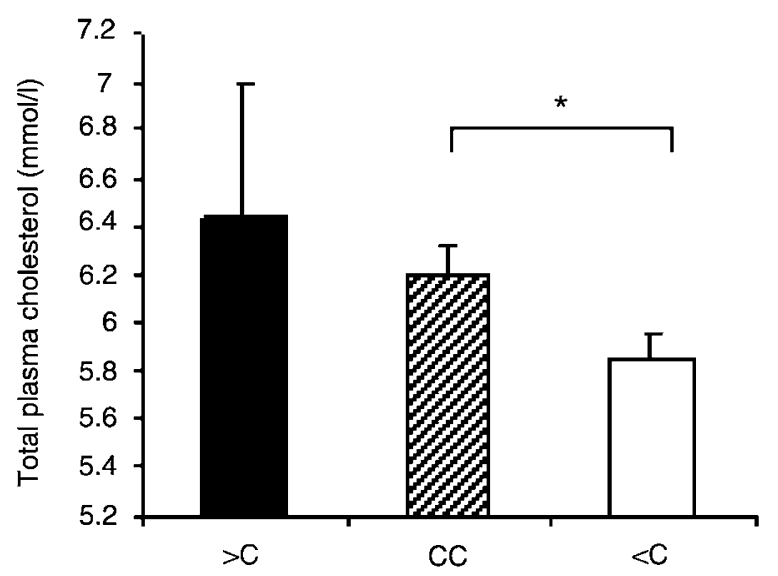

\begin{tabular}{|c|c|c|}
\hline genotype & cholesterol $(\mathrm{mmol} / \mathrm{l})$ & $\mathrm{n}$ \\
\hline$>\mathrm{C}$ & 6.4 & 10 \\
\hline $\mathrm{CC}$ & 6.2 & 112 \\
\hline <C & 5.8 & 108 \\
\hline
\end{tabular}

b

LDL

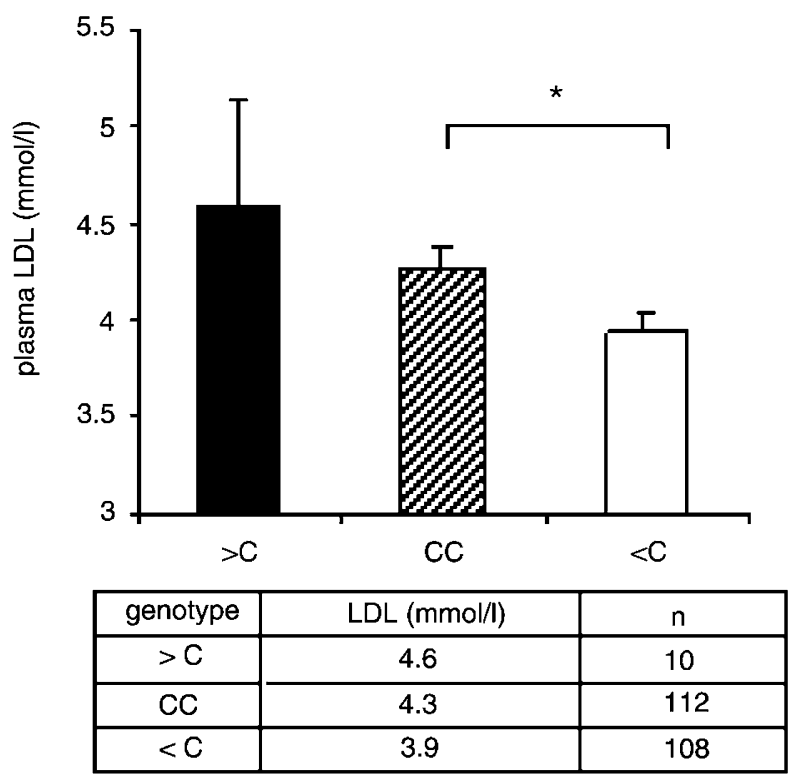

Figure 2 Differences in total cholesterol and LDL between genotype groups. Graphic illustration of the differences between the CC, $<\mathrm{C}$ and $>\mathrm{C}$ genotypes in the merged group (diabetes and control) with respect to total cholesterol (a) and LDL (b). Bars represent mean values \pm SE and ${ }^{*} P<0.05$.

shorter alleles confirmed that the deletions were within the region of the repeats. ${ }^{14}$ Another study later confirmed this diversity in the number of repeats and proceeded to compare the allele frequencies in a Caucasian and a
Japanese population. ${ }^{15}$ This latter study reported that $65 \%$ of the Caucasian subjects are heterozygous for alleles with 14 to 18 repeats in exon 11 of the CEL gene, while $44 \%$ of the Japanese subjects are heterozygous for alleles with 13 to 18 repeats. Although there was a tendency towards a difference in allele distribution between the populations, it did not reach statistical significance. ${ }^{15}$

The role of the repeats in CEL exon 11 is not clear, although there are indications to suggest that they may protect CEL from proteolytic degradation ${ }^{16}$ and that Oglycosylation of the repeated sequences could be important for the secretion of the enzyme. ${ }^{17}$ Some clues can be obtained from the results of investigations of a variant of CEL, the fetoacinar pancreatic protein (FAPP), which has been detected in human embryonic and fetal pancreas and in pancreatic tumoral cell lines. ${ }^{20,21}$ FAPP is identical to CEL except for a $330 \mathrm{bp}$ deletion in the C-terminal region, corresponding to repeats $5-14 .^{22}$ The recent findings of a substantial variation in the number of repeats in the CEL gene between individuals ${ }^{14,15}$ (the present study) raises the question of whether FAPP is in fact a naturally occurring isoform of CEL, rather than an oncofetal variant expressed in proliferating cells. In various human fetal tissues as well as in human embryos CEL is ubiquitously expressed. ${ }^{23}$ This could suggest that the expression of CEL is important in rapidly growing cells, for instance, during developmental stages but also in cancer cells. Compared to CEL, FAPP is poorly secreted ${ }^{24}$ and appears to be mainly associated with membranes $^{22}$ or in the cytosol, ${ }^{25}$ with only minute amounts being secreted. While in need of thorough investigation, it is plausible to speculate that the CEL repeat polymorphism may have implications for intracellular processing and secretion of CEL as well as protein stability. Therefore, the physiological significance of the CEL polymorphism needs to be addressed.

Although previously reported studies have suggested that there may be a relationship between CEL genotype and lipid profile phenotype, ${ }^{1,26}$ these studies were not designed to differentiate between CEL and the CEL pseudogene. One of the early rationales for suggesting a relationship between CEL and lipid profile is the fact that the human CEL gene maps to locus q34.3 on chromosome $9^{27}$ close to the ABO blood group antigen locus, which has been associated with lipid phenotype and cardiovascular disease. ${ }^{28}$ It is possible that the association between lipid profile and the $\mathrm{ABO}$ locus is due to a polymorphism in the CEL gene. ${ }^{27}$ The present study is the first to investigate association between a putatively functional variation in the CEL gene and lipid profile, and we found an association between shorter CEL alleles (ie fewer repeats than the common 16) and lower total cholesterol as well as lower LDL. A possible explanation for this observation could be that CEL proteins with fewer proline-rich repeats are secreted at a lower rate and/or are less well protected from degradation in the intestinal tract, and therefore less 
efficiently aid the hydrolysis and uptake of cholesterol dietary lipids. It is also possible that circulating CEL modulates serum lipid profile outside the intestinal tract. A study by Brodt-Eppley et $a l^{29}$ showed a positive correlation between levels of CEL and LDL in the plasma, while there appeared to be no correlation between CEL and HDL. Future studies are warranted to investigate whether the polymorphism in the repeated region of the CEL gene affects serum levels of circulating CEL. The result of the current study gives further support to the notion that CEL may be involved in determining the plasma lipid composition. Future experiments aimed at determining the functional effect of variable number of repeats, such as measuring the rate of CEL secretion or enzyme activity in individuals with different repeat number genotypes will help elucidate whether the repeats play a role in regulating CEL secretion and activity.

\section{Acknowledgements}

We are grateful to Lena Rosberg for technical assistance. This work was supported by grants from Fredrik and Inga Thuring Foundation. SH B-E is supported by the Swedish Foundation for Strategic Research via the National Network for Cardiovascular Research.

\section{References}

1 Hui DY: Molecular biology of enzymes involved with cholesterol ester hydrolysis in mammalian tissues. Biochim Biophys Acta 1996; 1303: $1-14$

2 Wang CS, Johnson K: Purification of human milk bile saltactivated lipase. Anal Biochem 1983; 133: 457-461.

3 Howles PN, Carter CP, Hui DY: Dietary free and esterified cholesterol absorption in cholesterol esterase (bile saltstimulated lipase) gene-targeted mice. J Biol Chem 1996; 271: $7196-7202$.

4 Weng W, Li L, van Bennekum AM et al: Intestinal absorption of dietary cholesteryl ester is decreased but retinyl ester absorption is normal in carboxyl ester lipase knockout mice. Biochemistry 1999; 38: 4143-4149.

5 Hui DY, Howles PN: Carboxyl ester lipase: structure-function relationship and physiological role in lipoprotein metabolism and atherosclerosis. J Lipid Res 2002; 43: 2017-2030.

6 Nilsson J, Blackberg L, Carlsson P et al: cDNA cloning of humanmilk bile-salt-stimulated lipase and evidence for its identity to pancreatic carboxylic ester hydrolase. Eur J Biochem 1990; 192: 543-550.

7 Reue K, Zambaux J, Wong $\mathrm{H}$ et al: cDNA cloning of carboxyl ester lipase from human pancreas reveals a unique proline-rich repeat unit. J Lipid Res 1991; 32: 267-276.

8 Lidberg U, Nilsson J, Stromberg K et al: Genomic organization, sequence analysis, and chromosomal localization of the human carboxyl ester lipase (CEL) gene and a CEL-like (CELL) gene. Genomics 1992; 13: 630-640.

9 Lidmer AS, Kannius M, Lundberg L et al: Molecular cloning and characterization of the mouse carboxyl ester lipase gene and evidence for expression in the lactating mammary gland. Genomics 1995; 29: 115-122.

10 Madeyski K, Lidberg U, Bjursell G, Nilsson J: Characterization of the gorilla carboxyl ester lipase locus, and the appearance of the carboxyl ester lipase pseudogene during primate evolution. Gene 1999; 239: 273-282.

11 Gjellesvik DR, Lorens JB, Male R: Pancreatic carboxylester lipase from Atlantic salmon (Salmo salar). cDNA sequence and computer-assisted modelling of tertiary structure. Eur J Biochem 1994; 226: 603-612.

12 Blackberg L, Hernell O: The bile-salt-stimulated lipase in human milk. Purification and characterization. Eur J Biochem 1981; 116: 221-225.

13 Stromqvist M, Hernell O, Hansson L et al: Naturally occurring variants of human milk bile salt-stimulated lipase. Arch Biochem Biophys 1997; 347: 30-36.

14 Lindquist S, Blackberg L, Hernell O: Human bile salt-stimulated lipase has a high frequency of size variation due to a hypervariable region in exon 11. Eur J Biochem 2002; 269: 759-767.

15 Higuchi S, Nakamura Y, Saito S: Characterization of a VNTR polymorphism in the coding region of the CEL gene. J Hum Genet 2002; 47: 213-215.

16 Loomes KM, Senior HE, West PM, Roberton AM: Functional protective role for mucin glycosylated repetitive domains. Eur J Biochem 1999; 266: 105-111.

17 Bruneau N, Nganga A, Fisher EA, Lombardo D: O-glycosylation of $\mathrm{C}$-terminal tandem-repeated sequences regulates the secretion of rat pancreatic bile salt-dependent lipase. J Biol Chem 1997; 272: $27353-27361$

18 Groop L, Forsblom C, Lehtovirta M et al: Metabolic consequences of a family history of NIDDM (the Botnia study): evidence for sexspecific parental effects. Diabetes 1996; 45: 1585-1593.

19 Nilsson J, Hellquist M, Bjursell G: The human carboxyl ester lipase-like (CELL) gene is ubiquitously expressed and contains a hypervariable region. Genomics 1993; 17: 416-422.

20 Escribano MJ, Imperial S: Purification and molecular characterization of FAP, a feto-acinar protein associated with the differentiation of human pancreas. J Biol Chem 1989; 264: $21865-21871$

21 Mazo A, Fujii Y, Shimotake J, Escribano MJ: Expression of fetoacinar pancreatic (FAP) protein in the pancreatic human tumor cell line BxPC-3. Pancreas 1991; 6: 37-45.

22 Pasqualini E, Caillol N, Panicot L et al: Molecular cloning of the oncofetal isoform of the human pancreatic bile salt-dependent lipase. J Biol Chem 1998; 273: 28208-28218.

23 Roudani S, Miralles F, Margotat A et al: Bile salt-dependent lipase transcripts in human fetal tissues. Biochim Biophys Acta 1995; 1264: $141-150$

24 Mas E, Abouakil N, Roudani S et al: Human fetoacinar pancreatic protein: an oncofetal glycoform of the normally secreted pancreatic bile-salt-dependent lipase. Biochem J 1993; 289 (Part 2): 609-615.

25 Verine A, Bruneau N, Valette A et al: Immunodetection and molecular cloning of a bile-salt-dependent lipase isoform in HepG2 cells. Biochem J 1999; 342 (Part 1): 179-187.

26 Aleman-Gomez JA, Colwell NS, Vyas K et al: Relationship of human pancreatic cholesterol esterase gene structure with lipid phenotypes. Life Sci 1999; 64: 2419-2427.

27 Taylor AK, Zambaux JL, Klisak I et al: Carboxyl ester lipase: a highly polymorphic locus on human chromosome 9qter. Genomics 1991; 10: 425-431.

28 George VT, Elston RC, Amos CI et al: Association between polymorphic blood markers and risk factors for cardiovascular disease in a large pedigree. Genet Epidemiol 1987; 4: $267-275$.

29 Brodt-Eppley J, White P, Jenkins S, Hui DY: Plasma cholesterol esterase level is a determinant for an atherogenic lipoprotein profile in normolipidemic human subjects. Biochim Biophys Acta 1995; 1272: 69-72. 